\title{
Über Lautäußerungen und ihr Verhältnis zur Sprache (am Beispiel von pff)
}

In ihrem Beitrag zu Interjektionen als Elemente „zwischen Lexem und Lautobjekt“" stellen Reber/Couper-Kuhlen (2010) unter anderem vier, für eine moderne Sprachwissenschaft ausgesprochen interessante Fragen:

- Welche Einheiten gehören zur Sprache?

- Was für ein System ist Sprache?

- Welche Rolle spielen prosodische sowie visuell-räumliche Modalitäten?

- Wie soll man Sprache modellieren oder untersuchen?

Sie stützen sich dabei vor allem auf Untersuchungen zum Englischen. Der folgende Aufsatz baut auf diesen Fragen und den von den beiden Autorinnen entwickelten Thesen auf. Im Mittelpunkt soll vor allem die Frage stehen, inwiefern eine dichotomische Trennung zwischen sprachlichen Einheiten einerseits und außersprachlichen Einheiten andererseits Sinn hat, wenn man Sprache nicht als von ihrer Verwendung unabhängiges und geschlossenes System beschreiben will.

In den letzten Jahren haben zahleiche Untersuchungen $\mathrm{zu}$ verschiedenen Sprachen vor allem im Bereich der Syntax darauf hingewiesen, wie wesentlich es für das Verständnis von Sprache ist, sie als Sprache „im Gebrauch“ zu betrachten. Dies trifft vor allem (aber nicht nur) auf mündliche Interaktion zu (siehe etwa Günthner 2009; Hagemann et al. (Hgg.) 2013; Imo 2013, 2016).

Anhand von exemplarischen Analysen zu einer nicht-vokalischen Lautäußerung im Deutschen möchte ich im Folgenden zeigen, dass eine dichotomische Trennung zwischen sprachlich und nicht-sprachlich problematisch ist und dass die Sprachwissenschaft nur gewinnen kann, wenn sie ihre Konzeption von „Sprache“ überdenkt.

\section{Am Rande der Sprache: Interjektionen}

Einheiten am Rande der Sprache sind in der schriftsprachlich orientierten Grammatik durchaus betrachtet und beschrieben worden. Erwähnt werden soll hier die klassische Kategorie der Interjektion, die zwar einerseits aus der Sprachbetrachtung ausgeschlossen wurde (,language begins where interjections end“; Müller 1862: 374), andererseits aber als eine der klassischen partes orationis zumindest am Rande immer wieder diskutiert wurde (zusammenfassend siehe 
Ehlich 2007). Formal unterscheidet man seit Wundt (1911) primäre und sekundäre Interjektionen. Aus semantischer Perspektive steht häufig der Gefühlsausdruck im Mittelpunkt, Interjektionen werden beschrieben als Ausdruck einer mentalen oder emotionalen Haltung. Syntaktisch bezeichnen sie etwas „Dazwischengeworfenes“ (siehe Bussmann 1990: 346), sind isoliert und stehen im Deutschen, ähnlich wie Diskursmarker, gewöhnlich an der Peripherie einer Äußerung (zusammenfassend Ameka 1992a; Nübling 2004).

Nübling (2004) versucht, deutsche Interjektionen prototypisch zu erfassen und sie mithilfe eines Katalogs von obligatorischen und fakultativen Merkmalen zu beschreiben. Somit will sie einerseits die Nähe zu Onomatopoetika, Inflektiven, Diskursmarkern oder anderen „Kurzformeln“ zeigen, andererseits das prototypisch Besondere der Interjektion herausstellen. Prototypische Interjektionen sind formal „exzentrisch“ (Trabant 1983: 74), was sich, mehr oder weniger ausgeprägt, in der phonetisch-prosodischen Realisierung (z.B. tonale Strukturen), in der phonologischen Struktur (z.B. die Präsenz von nicht zum Phonemsystem des Deutschen gehörende Schnalz-, Pfeif- oder Würglaute), in der Graphie (z.B. keine orthographische Normierung), in der Morphologie (z.B. lexikalische Opakheit) und in der Syntax (z.B. Holophrastik) zeigt. Funktional gesehen sind sie expressiv, nonreferenziell, monologisch und bilden einen Sprechakt. Diese Bestimmung von Interjektionen weist nun eine ganze Reihe von Problemen auf (siehe etwa Reber/Couper-Kuhlen 2010), die Ehlich (2007: 426) u.a. damit begründet, dass die Linguistik sie lange Zeit ignoriert hat und ihr somit das Handwerkszeug fehlt, um sie $\mathrm{zu}$ beschreiben.

Ein Problem betrifft die funktionale Abgrenzung. Während einige Autoren explizit den Emotionsausdruck als primär oder zumindest prototypisch (Nübling 2004; Stange 2016) ansehen und somit Interjektionen von Diskursmarkern unterscheiden, weisen andere mehr oder weniger explizit auf andere Funktionen von Interjektionen hin: Ameka (1992b) etwa beschreibt phatische und konative Interjektionen, Norrick (2009) analysiert Interjektionen als ,pragmatic markers“ und zeigt vor allem ihre Funktionen in der Gesprächsorganisation auf und Ehlich (2007) argumentiert u.a., dass die Definition als „Gefühlswort“ leicht dazu führt, dass auch Flüche und andere „Gefühlsausdrücke“, die syntaktisch nicht wie eine Interjektion funktionieren, als Interjektion kategorisiert werden.

Zum anderen wird die kategoriale Abgrenzung als problematisch angesehen:

Die spezifische Differenz der interjektionellen Form gegenüber dem bezugssprachlich Typischen bzw. Allgemeinen lokalisiert diese Form in einem Bereich, in dem sich auch andere akustisch-formale Phänomene befinden [...]. Einige davon sind sprachlicher Art, einige sind interaktionell-kommunikativer, also nichtsprachlicher Art. (Ehlich 2007: 427) 
Die Frage nach der Sprachlichkeit und der Abgrenzung zu verwandten Formen wie Onomatopoetika, Diskursmarkern, Routineformeln etc. einerseits und nichtkonventionellen Lautäußerungen wie Lachen, Seufzen, Stöhnen oder Quieken andererseits wird überwiegend ohne Bezugnahme auf die tatsächliche Verwendung der untersuchten Form in (mündlichen oder schriftlichen) Interaktionen diskutiert. Insofern basiert die Argumentation vor allem auf der semantischpragmatischen Beschreibung von (bestimmten Arten von) Interjektionen (z.B. Wilkins 1992; Wharton 2003; Meinard 2015), andere Aspekte wie etwa die Sequenzialität werden außer acht gelassen bzw. als „parasprachlich“ betrachtet. In Bezug auf die „Parasprachlichkeit“ von Interjektionen wird u.a. auf Goffman (1981) verwiesen, der aus interaktionaler Perspektive sogenannte response cries zunächst als „nonwords“ (ebd.: 115) bezeichnet und meint, „nonwords [...] can’t quite be called part of a language“ (ebd.). Im weiteren Verlauf argumentiert er aber für eine differenziertere Sicht:

Yet the sound that covers any particular nonword can stand by itself, is standaradized within a given language community, and varies from one language community to another, in each case as do full-fledged words. (ebd.: 115f.)

Goffman spricht daraufhin von „semiwords“ (ebd.: 116), Elementen, die einerseits nicht dem traditionellen Verständnis von Sprache entsprechen, andererseits aber doch mehr oder weniger sprachgebunden sind.

Das Problem der Sprachlichkeit stellt sich nun zum einen aufgrund der Verschiedenheit der als Interjektion bezeichneten Formen - selbst wenn man nur die primären Interjektionen berücksichtigt, findet man etwa unter den Items, die Pompino-Marschall (2004: 76) als Interjektionen identifiziert, eindeutig lexikalisierte Formen wie „Mensch“ neben weniger eindeutig lexikalisierten Formen wie „(p)ff“. Formal gesehen würde sich demnach für mache Interjektionen die Frage der Sprachlichkeit nicht stellen, für andere aber schon. Zudem handelt es sich, folgt man Norrick (2009), bei Interjektionen um eine offene, unbegrenzte Klasse, und der Übergang zu anderen Lauten ist fließend: „Any of these sounds may function as an interjection [...]“ (ebd.: 869). Von einem fließendem Übergang, einem Kontinuum ist auch an anderer Stelle die Rede. So geht PompinoMarschall (2004) in seinen Untersuchungen zur Phonetik der Interjektion von einem Kontinuum zwischen unartikuliertem Naturlaut (in Anlehnung an Wundt 1911) und „kanonisch korrekter Aussprache von Inhaltswörtern“ (ebd.: 71) aus. Wharton (2003) spricht aus referenzlinguistischer Sicht von einem Kontinuum zwischen showing und saying, von ,sounds which are essentially used to show, but have an extra layer of encoding which puts them on the borderline of saying“ (ebd.: 78). 
Zum anderen ist das Problem mit der Sprachlichkeit der Interjektionen darauf zurückzuführen, dass man versucht, die Sprachlichkeit unabhängig vom Gebrauch zu definieren und etwa die Bedeutung einer Interjektion kontextunabhängig zu beschreiben (siehe etwa Wilkins 1992; Wharton 2003; Goddard 2013). Nun hat aber die interaktionale Linguistik in den vergangenen zwei Jahrzehnten eindrücklich gezeigt, wie wichtig es für das Verständnis von sprachlichen Strukturen ist, diese in ihrem sequenziellen Kontext zu untersuchen (siehe etwa Selting/Couper-Kuhlen 2000). Mit dem Blick auf Sprache-in-Interaktion wurde deutlich, dass die „gesprochene“ Sprache nicht nur genauso musterhaft, geordnet und geregelt ist wie die „geschriebene“, sondern auch, dass grammatische Strukturen in der (mündlichen) Interaktion ,heterogener, offener und kontextabhängiger" (Günthner 2009: 403) sind. Die Besonderheiten mündlicher Interaktion wie „Dialogizität, inkrementelle Entfaltung sprachlicher Strukturen, Zeitlichkeit ihrer Produktion etc." (ebd.: 419) wurden in traditionellen linguistischen Theorien aufgrund der written language bias (Linell 2004) häufig nicht oder nur unzureichend zur Kenntnis genommen und typisch gesprochensprachliche Muster als „abweichend“ charakterisiert (zusammenfassend Selting 2007; Imo 2013). Das trifft nun auch auf Interjektionen oder Lautäußerungen als „Wortklasse“ zu. Es zeigt sich, dass die Schwierigkeiten mit der Beschreibung von Interjektionen nämlich zu einem großen Teil auf dem fehlenden „Handwerkszeug“ beruhen, wie Ehlich (2007: 426) so treffend bemerkt. Betrachtet man sie „holistischer und realistischer“ (Günthner 2009: 422), berücksichtigt man einerseits ihre sequenzielle Einbettung und andererseits ihre phonetische und materielle Realisierung, d.h. ihre Körperlichkeit, verlieren die Schwierigkeiten an Bedeutung. Angeführt seien hier etwa die Untersuchung von Reber (2012) zu (englischem) „oh“ bzw. Barth-Weingarten (2011) zu (deutschem) ,jaja“. Beruft man sich auf solche Studien, stellt sich die Frage nach der Sprachlichkeit auf eine andere Art und Weise: Anstelle zu suchen, ob und wie Interjektionen in unser (auf der Reflexion über Schriftsprache beruhendes) Verständnis von Sprache passt, wäre zu überlegen, wie unser Verständnis von Sprache dem tatsächlichen Sprachgebrauch in der (mündlichen) Interaktion angepasst werden kann. Ähnliches trifft sicher auch auf schriftliche Interaktion zu, sobald man sie als situiert (im Sinne von Goodwin 2000) und dialogisch (Imo 2016) betrachtet. 


\section{Ein Beispiel: Die Lautäußerung pff im Deutschen}

\subsection{Ausgangspunkt}

Vor dem Hintergrund dieser Überlegungen möchte ich nun eine sehr frequente, aber kaum untersuchte Lautäußerung näher betrachten. Im Rahmen einer (phonetischen) Analyse eines fünfundvierzigminütigen Fernsehinterviews listet Pompino-Marschall (2004) eine Reihe von 83 „'echten' Interjektionen“ (ebd.: 76) entsprechend der Häufigkeit ihres Vorkommens. An dritter Stelle, mit einer Auftretenshäufigkeit von 12\%, rangiert dabei eine Interjektion, die er als „(p)ff“ angibt. Diese Interjektion ist, so der Autor, durch eine grundsätzlich defekte phonologische Lautstruktur (kein Vokal, ebd.: 72) gekennzeichnet und kann auf verschiedene Arten realisiert werden: Es werden u.a. Realisierungen mit bilabialem Trill, stimmlosem bilabialem Frikativ und glottalem Stop angegeben. Insgesamt listet Pompino-Marschall zehn verschiedene Aussprachevarianten, von denen er einige einer bestimmten Verwendungsweise zuordnet, nämlich dem „Zitieren“ im Rahmen einer direkten Redewiedergabe. Außerdem weist er auf die unterschiedliche Länge der Interjektion hin, die von 110 bis 675 Millisekunden dauern kann - jeweils in Abhängigkeit von der phonetischen Realisierung. Trotz ihrer Häufigkeit in dem analysierten Interview findet man die Interjektion „(p)ff“ jedoch nicht in der Liste potenziell prototypischer Interjektionen von Nübling (2004) - wobei allerdings nicht klar ist, wie diese Liste entstand. Da gibt es aber eine Interjektion „phh!“, die Gleichgültigkeit und Verachtung ausdrücken soll. Sucht man in einschlägigen Wörterbüchern des Deutschen, findet man zwar vereinzelt Beispiele schriftlicher Verwendung einer Interjektion „pff“ oder „pph“, jedoch keinen Eintrag zu „pf“, „pff“, „ff“ oder „pph“. Das Lehr- und Forschungskorpus für gesprochenes Deutsch (FOLK) des Instituts für Deutsche Sprache verzeichnet allerdings eine Reihe von Okkurrenzen, die als „pff“ (228), „pf“ (79) oder „ph“ (22) transkribiert werden, wobei darunter jeweils verschiedene phonetische Varianten zusammengefasst sind.

Es scheint also, dass so etwas wie „pff“ als Lautäußerung existiert, jedoch in der Forschung nicht oder kaum als Interjektion wahrgenommen wird. Dies mag sowohl Grund als auch Folge einer sehr unterschiedlichen Schreibung sein, wobei einige der von Pompino-Marschall beschriebenen Varianten (etwa mit einem bilabialen Trill oder einem stimmlosen bilabialen Frikativ) graphisch nicht wiedergegeben werden können, da diese Laute nicht zum Phonemsystem des Deutschen gehören und keine graphische Entsprechung im Deutschen haben. 
Wie problematisch dies unter Umständen ist, zeigen die Angaben zur Transkription von „Interjektionen“ (Schmidt/Schütte/Winterscheid 2015: 21f.).

Aber auch im Rahmen der interaktionalen Linguistik gibt es kaum Untersuchungen. Dies lässt sich sicher auch auf die Randstellung von „pff“ als nichtvokalische Lautäußerung zurückführen, die verschieden realisiert wird, verschiedene Funktionen erfüllen kann, an verschiedenen sequenziellen und Turnpositionen auftritt und wohl relativ häufig mit anderen Partikeln bzw. Lautäußerungen kombiniert wird.

Interessant ist, dass es eine als „pf“/“pff“ umschriebene Lautäußerung auch in anderen Sprachen gibt. Allerdings scheint es mir zu voreilig, davon auf eine mögliche Universalität oder Natürlichkeit zu schließen. Meine eigenen Untersuchungen zum Französischen (Baldauf-Quilliatre 2016, 2017) haben gezeigt, dass es sowohl in der Verwendung, als auch in der Realisierung Überschneidungen und Unterschiede gibt. Dies deutet darauf hin, dass „pff“ möglicherweise näher an „natürlichen Lauten“ (im Sinne von Darwin 1873 bzw. Trojan 1948) ist, bedeutet aber gleichzeitig, dass es sich um eine sprachlich überformte und daher sprachspezifische Weiterentwicklung handelt. Darauf soll aber in diesem Rahmen nicht weiter eingegangen werden (siehe Baldauf-Quilliatre i.Vorb.).

\section{2 pff im Gebrauch}

Eine erste Annäherung an die Lautäußerung im Korpus FOLK des Instituts für Deutsche Sprache Mannheim zeigt, dass sie in verschiedenen sequenziellen und Turnpositionen auftritt. So findet man „pff“ freistehend, turn-initial, turnfinal, zu Beginn oder am Ende einer Interaktionseinheit, und zum großen Teil im Rahmen von Frage- oder Erklärsequenzen.

Die folgenden vier Beispiele zeigen fünf verschiedene Lautäußerungen (alle jeweils als „pff“ transkribiert) in verschiedenen sequenziellen Umgebungen. Ich möchte im Weiteren verdeutlichen, dass allein die Berücksichtigung der sequenziellen Umgebungen verschiedene Funktionen sichtbar macht, die sich nur schwer unter einem Begriff zusammenfassen lassen. ${ }^{1}$

1 Auf Unterschiede in der phonetisch-prosodischen Realisierung bzw. auf Aspekte der Körperlichkeit wird hier nicht näher eingegangen (siehe Baldauf-Quilliatre i.Vorb.). Daher handelt es sich bei den Transkriptionen jeweils auch nur um ein Minimaltranskript (mit Ausnahme des „pff“-Turns). 
Beispiel 1: Spielinteraktion (Monopoly) FOLK_E_00011_SE_01_T_03_DF_01

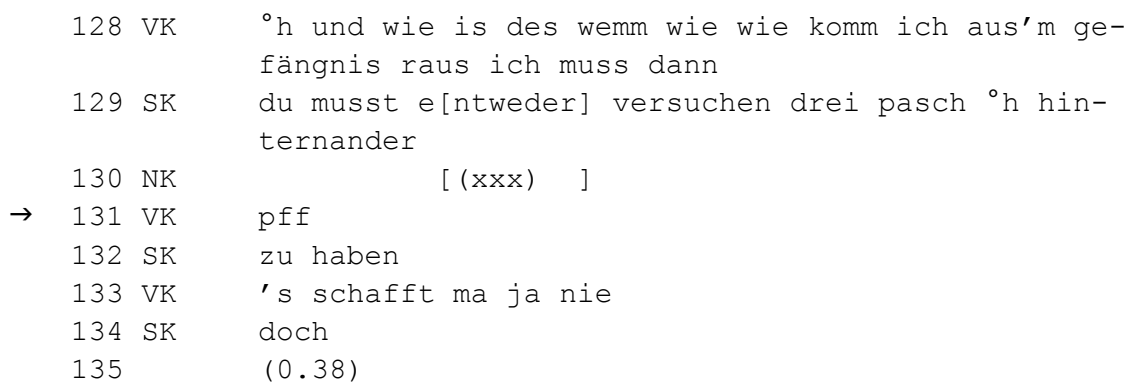

Das erste Beispiel stammt aus einer Interaktion zwischen einem Vater und seinen beiden Töchtern (6 und 9 Jahre alt) beim gemeinsamen Monopoly-Spielen. In diesem Ausschnitt stellt der Vater eine Frage hinsichtlich einer Spielregel (Z. 128). Die ältere Tochter Sabine antwortet ihm und erklärt, was er in der vorliegenden Situation tun muss, nämlich drei Pasch hintereinander würfeln (Z. 129 und 132). Der Vater kommentiert nun diese Möglichkeit zunächst mit einer Lautäußerung und anschließend mit einer Bewertung als zu schwierige, unlösbare Aufgabe (Z. 131). Die Lautäußerung „pff“ (ein stark artikulierter stimmloser labiodentaler Frikativ von 0.2 Sekunden) kommt hier sicher am ehesten der expressiven Kundgabe-Funktion einer Interjektion bzw. dem response cry im Sinne Goffmans nahe: als „presumed 'natural expressions', [...] meant to be taken to index directly the state of the transmitter“ (Goffman 1981: 116), die keine spezifische Nachfolgehandlung projiziert (ebd.: 108). Sie ist rückwärtsgerichtet und zeigt eine emotionale Reaktion in Hinblick auf Sabines Antwort. Allerdings spezifiziert der Vater diese erste Reaktion im folgenden Turn durch eine Bewertung. Brès (1995: 86ff.) zeigt, allerdings für schriftliche Texte, dass (französische) Interjektionen regelmäßig einer verbalen Äußerung vorausgehen (,interjection comme étape initiale de l'actualisation phrastique“). Ähnlich argumentieren auch Kowal/O'Connell (2004: 97) in Hinblick auf die Verwendung von (deutschen) Interjektionen in einem Fernsehinterview. Für dieses Beispiel bedeutet das: Lautäußerung und Bewertung sind nicht getrennt $\mathrm{zu}$ betrachten, gemeinsam können sie als preliminary cues einer Klage angesehen werden (siehe Traverso 2009), die Sabine jedoch zurückweist, indem sie mit einem dispräferierten gegenläufigen „doch“ widerspricht.

Eine vergleichbare Verwendung von „pff“ findet sich auch im zweiten Beispiel, einem Ausschnitt aus einer Spielinteraktion zwischen Erwachsenen: In diesem Ausschnitt erklärt Pascal, der Gastgeber des Spieleabends, den anderen Mitspielern und Mitspielerinnen die Spielregeln des Brettspiels „Thurn und Taxis“ (Zeile 434-442). Diese kommentieren die Erklärung auf unterschiedliche Weise. 
Beispiel 2: Spielinteraktion (Thurn und Taxis) 151

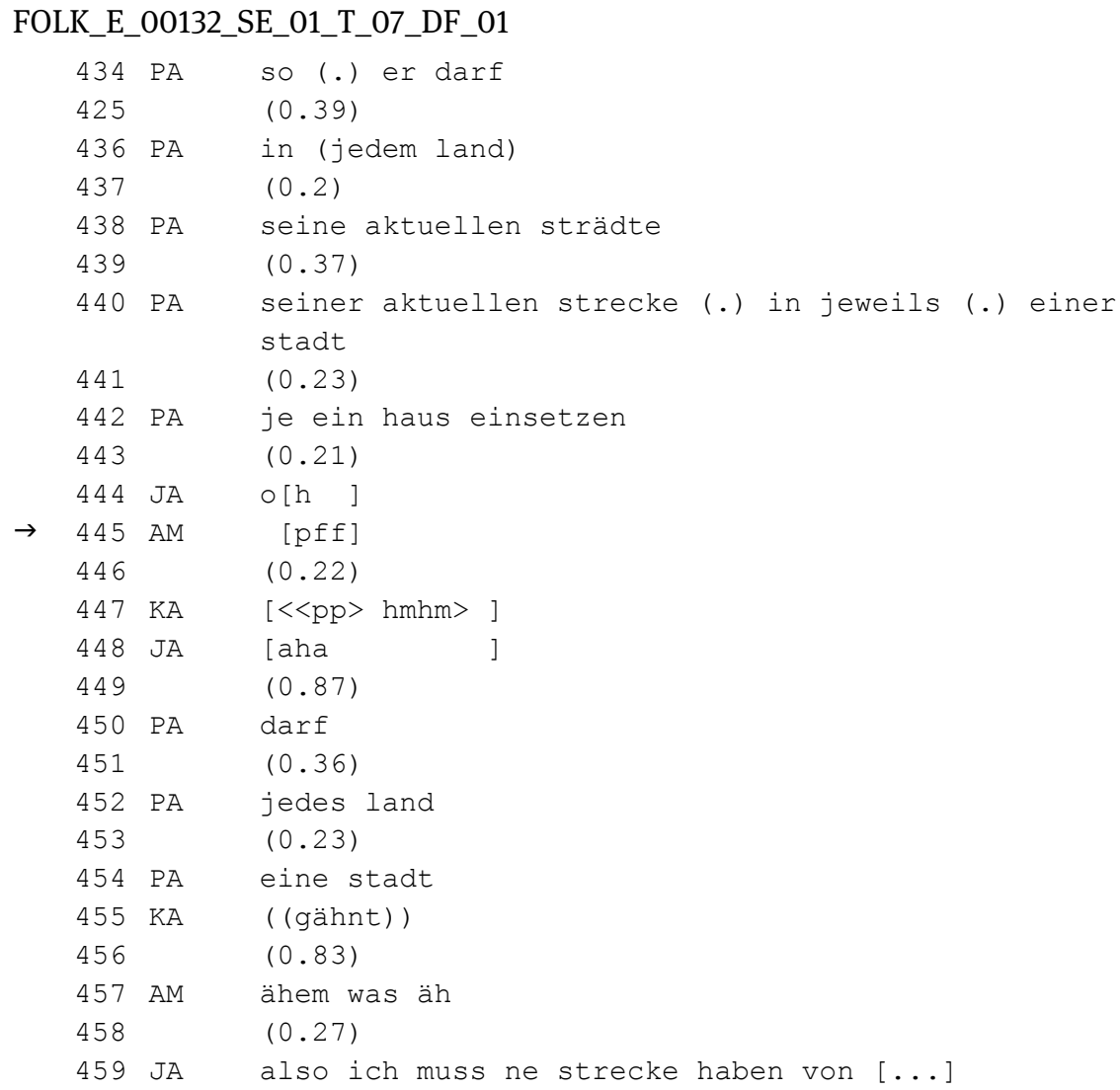

Jan zeigt an, dass er eine neue Erkenntnis gewonnen hat (Z. 444), wobei „oh“ im Deutschen dazu dient, eine gewisse Emotionalität in Hinblick auf diese neue Erkenntnis deutlich zu machen (Golato 2012). Amanda äußert fast gleichzeitig „pff“ (einen stimmlosen bilabialen Trill von 0.47 Sekunden, Z. 445) und Katja gibt nach einer kurzen Pause mit einem leisen reduplizierten „hm“ (Z. 447) an, dass sie verstanden hat (Ehlich 1986). Pascal fährt nach einer längeren Pause mit seiner Erklärung fort und präzisiert die Regel (Z. 450-454). Jetzt unterbricht ihn Katja mit einem Gähnen und Amanda fragt nach einer Pause. Jan versucht nun nach seinem Verständnis die Spielregel zu erklären (Z. 459ff.). Während Jan durch verschiedene Erkenntnisprozessmarker („oh“, „aha“) und die Übernahme der Rolle des Erklärers sowohl sein Verstehen als auch sein Engagement im Verstehensprozess anzeigt, machen Amanda und Katja deutlich, dass sie weniger bei der Sache sind und Mühe haben, den Erklärungen zu folgen. Ähnlich wie in Bei- 
spiel 1 ist Amandas Lautäußerung rückwärts gerichtet. Allerdings erfolgt sie erst am Ende einer interaktionalen Einheit im Rahmen einer relativ komplexen Erklärung und sie bezieht sich weniger auf den Inhalt des vorangegangenen Turns. Zudem steht sie allein. Amandas „pff“ stellt somit eine Art receipt token dar, das einerseits anzeigt, dass die Sprecherin die Erklärung als solche quittiert und das andererseits, ähnlich wie Seufzen, eine (eher negative) emotionale Einstellung in Bezug auf diese Erklärung äußert (Hoey 2014: 186).

Als receipt token kündigt Amandas Lautäußerung keine Übernahme des Rederechts an und projiziert auch keine spezifische Antworthandlung. In den nachfolgenden Turns wird daher auch nicht darauf Bezug genommen und Pascal setzt seine Erklärung fort.

Dies ist anders im nächsten Beispiel.

Beispiel 3: Sprachbiografisches Interview FOLK_E_00129_SE_01_T_01_DF_01

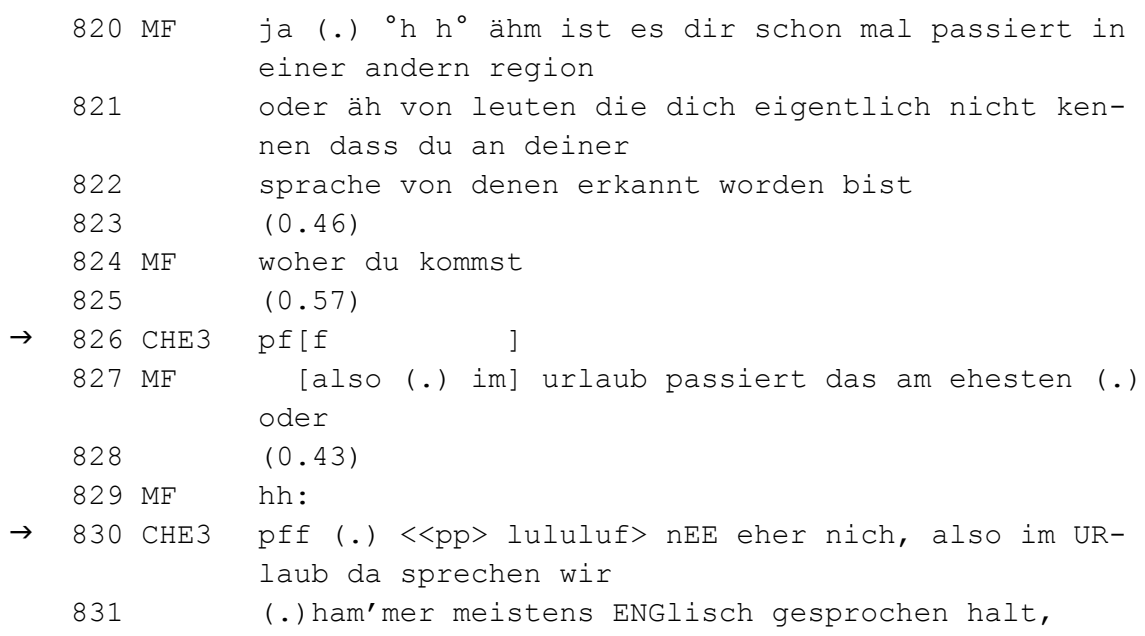

Das Beispiel stammt aus einem sprachbiografischen Interview mit einem Gymnasialschüler in Obersachsen. In dem Ausschnitt fragt der Interviewer Markus den Schüler CHE3, ob man ihn schon anderswo an seiner „sprache“ (seinem Dialekt) als Sachse „erkannt“ hat (Z. 820-822). Da CHE3 nicht sofort antwortet, präzisiert der Interviewer seine Frage (Z. 824). Auch hier antwortet CHE3 nicht sofort. Erst nach einer Pause von 0.57 Sekunden erfolgt die Lautäußerung „pff“ (Z. 826; ein stimmloser bilabialer Plosiv und ein stimmloser bilabialer Frikativ von 0.77 Sekunden), die allerdings keine ausreichende Antwort auf die Frage nach der Identifizierung als Sachse darstellt. Der Interviewer präzisiert daher im Overlap noch einmal seine Frage, indem er ein Antwortangebot (Pomerantz 1988, 2012) 
liefert (Z. 827). Pomerantz (1988: 367) sieht eine Funktion für Antwortangebote darin, dem Befragten ein Modell zu geben, ihn bei seiner Antwort zu führen, u.a. „when an interactant anticipates or observes a co-interactant having difficulty giving a satisfactory answer without a model“. Nach einer weiteren Pause antwortet der Interviewte schließlich mit einer zögerlichen Verneinung, die wiederum durch ein „pff“ (stimmloser bilabialer Plosiv und stimmloser bilabialer Frikativ von 0.58 Sekunden) eingeleitet wird (Z. 830-831).

Im Gegensatz zu Beispiel 1 und 2 handelt es sich hier bei beiden Lautäußerungen weder um ein receipt token, noch um die Kundgabe einer negativen affektiven Einstellung. Als erster Teil einer Paarsequenz projiziert Markus' Frage eine Antwort als zweiten Teil. Sowohl die Pausen, als auch die Präzisierungen und Antwortangebote des Fragenden und die zögerliche Antwort in Zeile 830 deuten darauf hin, dass „pff“ hier wohl eher Unsicherheit bzw. Schwierigkeiten beim Beantworten der Frage anzeigt: In Zeile 826 verzögert „pff“ die Antwort und der Interviewer reagiert auf diese Verzögerung, indem er Hilfestellung leistet. In Zeile 830 kündigt „pff“ am Turnbeginn an, dass die Beantwortung trotz der Hilfestellung schwierig bleibt. Dieses zweite „pff“ lässt sich insofern als Einstellungsmarker (Baldauf-Quilliatre 2017) interpretieren.

Während in den bisher diskutierten Beispielen „pff“ nach einem Sprecherwechsel, genauer gesagt nach einer Erklärung bzw. einer Frage, am Turnbeginn (Beispiel 1 und 3) oder freistehend (Beispiel 2) verwendet wurde, steht „pff“ im nächsten Beispiel am Anfang einer neuen Interaktionseinheit des gleichen Sprechers.

Beispiel 4: Tischgespräch unter Studentinnen

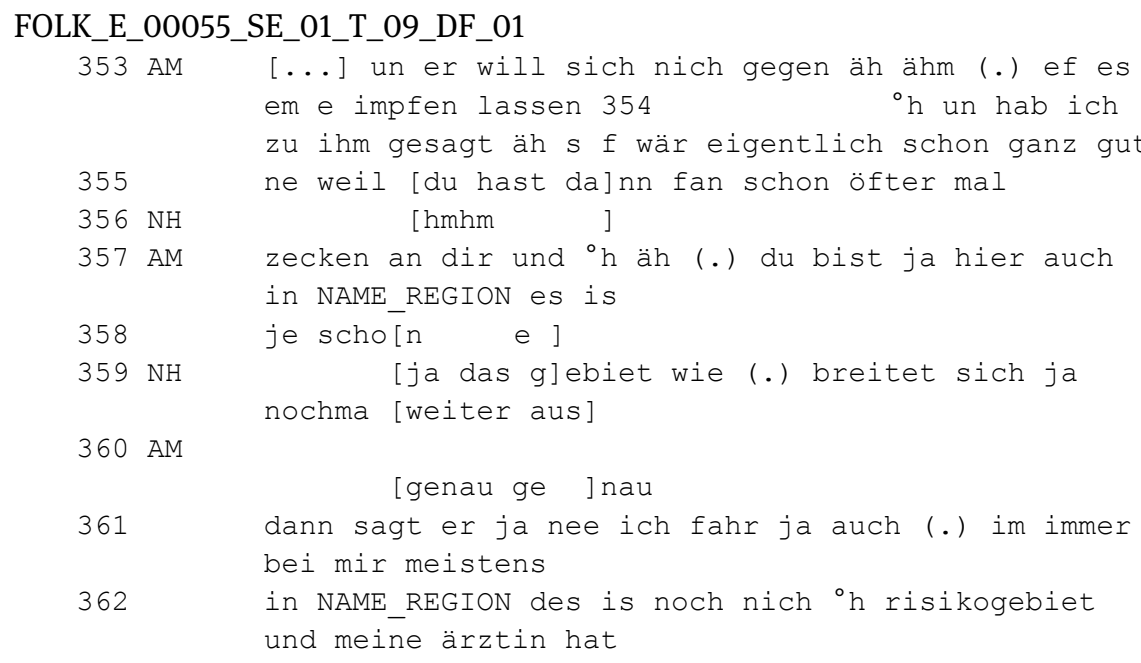




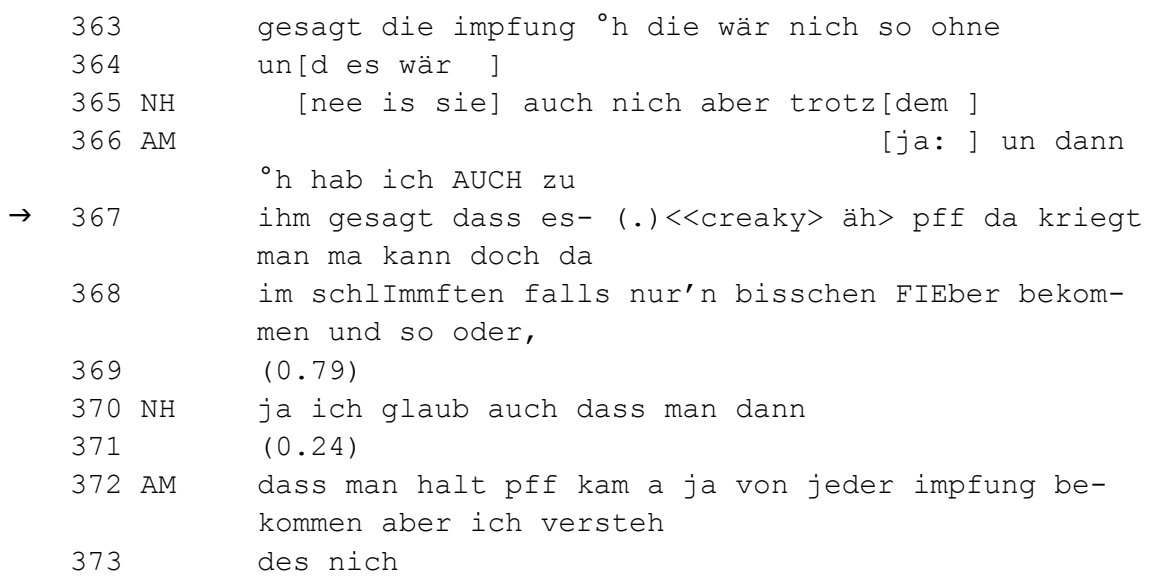

Der Ausschnitt stammt aus einem Tischgespräch zwischen vier Studentinnen in einer Wohngemeinschaft. Anita erzählt in einer längeren Phase von ihrem Freund, der sich nicht gegen FSME (Frühsommer-Meningoenzephalitis) impfen lassen will, eine Entscheidung, die sie augenscheinlich nicht versteht und nicht gutheißt. In einer Redewiedergabe (Z. 359-362) gibt sie Gründe an, mit denen ihr Freund die Impfung ablehnt. Einer der Gründe ist dabei der Hinweis auf die Information seitens einer Ärztin, die Impfung sei nicht ungefährlich. An dieser Stelle unterbricht Nicola und bestätigt die Information, leitet jedoch gleichzeitig ein Gegenargument ein (,aber trotzdem“, Z. 365). Anita unterbricht nun ihrerseits Nicola und nimmt ihre Erzählung mit einer indirekten Redewiedergabe ihrer eigenen Gegenargumente wieder auf. Sie beginnt eine dass-Konstruktion mit einem Matrixsatz („dann hab ich auch zu ihm gesagt“) und einem mit dass eingeleiteten Komplementsatz („dass es“), bricht allerdings ihre dass-Konstruktion ab und beginnt eine neue Konstruktion mit Verbzweitstellung („da kriegt man“). Auch diese Konstruktion bricht sie ab und setzt mit einer neuen Konstruktion an („ma kann doch da [...] bekommen"), die sie zu Ende führt und mit einem question tag abschließt (Z. 366-368).

Während Mikropause und Zögerungspartikel verbunden mit dem Wechsel in die Knarrstimme (Z. 367) als Anzeichen für Unsicherheit und die Suche nach der geeigneten Konstruktion interpretiert werden können, stellt „pff“ (ein stimmloser labiodentaler Frikativ von 0.47 Sekunden) eher einen Neubeginn dar und ist als Vorlauf (Schegloff 1996) der nachfolgenden Einheit zu verstehen. Die Sprecherin wechselt hier wieder in ihre normale Sprechstimmlage zurück und schließt ohne Unterbrechung eine neue Konstruktion an, die durch den question tag rückwirkend einen Wechsel aus der Redewiedergabe in die direkte Interaktion mit den anderen Gesprächsteilnehmerinnen anzeigt. In Bezug auf diesen Wechsel kann 
die Lautäußerung „pff“ hier als Einstellungsmarker interpretiert werden, der den sprachlich explizierten Widerspruch gegenüber den zuvor wiedergegebenen Argumenten von Anitas Freund einleitet.

\section{Diskussion}

Betrachtet man die vier Beispiele, lässt sich zunächst Folgendes feststellen:

In Beispiel 1 handelt es sich um einen response cry, der nicht am Ende einer interaktionalen Einheit geäußert wird. Nach dem response cry erfolgt - am Ende der Einheit - eine Spezifizierung durch denselben Sprecher (Ankündigen einer Klage). Der erste Sprecher unterbricht daraufhin seine Erklärung und antwortet auf die Klage.
A: Erklä[rung]
B: $\quad[p f f]+$ Spezifizierung
A: Antwort

In Beispiel 2 stellt die Lautäußerung ein receipt token nach einer Erklärung dar, mit dem kein Rederecht beansprucht wird; der erste Sprecher setzt seinen Turn fort. Als receipt token könnte „pff“ in diesem Fall im Overlap geäußert werden, auch wenn dies in Beispiel 2 nicht der Fall ist.
A: $\quad$ Erklärung
B: $\quad p f f$
A: Weiterführen der Erklärung

In Beispiel 3 erfolgt die erste Lautäußerung nach einer Frage und zeigt eine sich verzögernde Antwort an. Der Fragende kann in diesem Fall seine Frage präzisieren, erklären oder andere Hilfestellungen geben (etwa durch ein Antwortangebot wie hier); dies ist aber nicht immer der Fall.
A: $\quad$ Frage
B: $\quad p f f$
[A: Präzisieren, Explizieren, Reformulieren der Frage]
B: Antwort

Die zweite Lautäußerung steht am Turnbeginn und leitet die Antworthandlung ein. Auch sie weist auf Schwierigkeiten bei der Beantwortung der Frage hin. 

A: $\quad$ Frage
B: $\quad p f f+$ Antwort

In Beispiel 4 erscheint die Lautäußerung im Turn eines Sprechers als Vorlauf einer neuen Interaktionseinheit, nach einem Konstruktionsabbruch und verschiedenen Zögerungsphänomenen. Sie funktioniert hier als emotionaler Einstellungsmarker, der die nachfolgende Einheit in Bezug setzt zum vorher Gesagten.

A: Interaktionseinheit 1 - Zögerungsphänomene - pff - Interaktionseinheit 2

Und nicht nur hinsichtlich der sequenziellen Einbettung unterscheiden sich die fünf Beispiele. Während „pff“ in Beispiel 1, 2 und 4 eher eine Einstellung des Sprechers anzeigt, handelt es sich in Beispiel 3 vor allem um das Markieren eines Problems mit dem Frage-Turn. CHE3 zeigt, dass er weiß, was von ihm erwartet wird (nämlich der projizierte zweite Teil der Paarsequenz), dass er diesen zweiten Teil jedoch momentan nicht liefern kann (Z. 826) bzw. dass er Schwierigkeiten hat, ihn zu produzieren (Z. 830).

Betrachtet man nun die drei Beispiele, in denen „pff“ eine Einstellung markiert, näher, fallen auch hier signifikante Unterschiede auf: In Beispiel 1 und 2 bezieht sich „pff“ auf die Erklärung eines vorangegangenen Sprechers. Der Sprecher äußert damit eine Einstellung hinsichtlich des Inhalts der Erklärung (Beispiel 1: „was man von mir verlangt, ist zu schwer“) oder hinsichtlich der Erklärung als solche (Beispiel 2: „die Erklärung ist zu kompliziert/unverständlich/ nicht nachvollziehbar“). In Beispiel 4 hingegen hat „pff“ eine Scharnierfunktion zwischen zwei Interaktionseinheiten desselben Sprechers. Es verwirft die in der ersten Interaktionseinheit aufgeführten Argumente als ,unwesentlich“ und kündigt den in der zweiten Interaktionseinheit explizierten Widerspruch an (vergleichbar dem Anzeigen eines Orientierungswechsels, Baldauf-Quilliatre 2016).

Beispiel 1 und 2 scheinen auf den ersten Blick ähnlich. Während jedoch in (1) „pff“ eine Einstellung äußert, die sowohl rückwärtsgewandt als emotionale Kundgabe auf die Erklärung bezogen ist, als auch vorwärts gerichtet zum Verständnis der Klage beiträgt, ist „pff“ in (2) im Rahmen von verschiedenen receipt tokens verschiedener Sprecher zu sehen.

Deutlich wird an diesen vier Ausschnitten Folgendes:

1. Bei der Interpretation aller Beispiele (vielleicht mit Ausnahme von Beispiel 1, das sich am ehesten als response cry interpretieren lässt und bei dem wohl am ehesten die Funktion der emotionalen Kundgabe im Mittelpunkt steht) werden interaktionale Kategorien mobilisiert, die man üblicherweise für die Beschreibung eher sprachlicher Phänomene benutzt. Dies widerspricht nun 
allerdings der phonetisch motivierten Einschätzung von Pompino-Marschall (2004), der „pff“ zu den sogenannten „wilden“ Interjektionen zählt, die er in Anlehnung an Trubetzkoy (1939) als „Stimmgebärden“ am „Übergang zu parasprachlichen, nicht-konventionellen Lautäußerungen“ (Pompino-Marschall 2004: 73) bezeichnet.

2. Von einer emotionalen Kundgabe kann man, zumindest in Beispiel 3 und 4, nicht sprechen. Dies ist aber in den meisten Studien die Hauptfunktion prototypischer Interjektionen. Sollte man also tatsächlich Interjektionen wesentlich weiter fassen? Wo aber befindet sich dann die Grenze zu Diskursmarkern?

3. Obwohl es sich um einen Laut handelt, der in allen vier Beispielen gleich transkribiert wurde (nämlich als „pff“ - mit Ausnahme des zweiten „pff“ in Beispiel 3), handelt es sich keinesfalls um die gleiche Lautäußerung. Sowohl hinsichtlich der sequenziellen Einbettung, als auch hinsichtlich der Semantik und Pragmatik gibt es deutliche, aber systematische Unterschiede.

\section{Fazit}

Interjektionen sind eine schillernde Kategorie, unter der eine Reihe sehr unterschiedlicher Elemente zusammengefasst werden. Verschiedene Arbeiten weisen immer wieder darauf hin, dass sie sich vor allem durch ihre Verschiedenheit, sowohl in der Form, als auch im Gebrauch auszeichnen. Dem muss auch in der Untersuchung Rechnung getragen werden: Interjektionen kann man meines Erachtens nicht bestimmen, ohne ihren Gebrauch in tatsächlichen Interaktionen zu analysieren. Dies bedeutet aber auch, dass die Interaktion als solche ernst genommen werden muss. Eine Unterscheidung zwischen interaktionalen Phänomenen einerseits und sprachlichen Phänomenen andererseits macht bei einem solchen Vorgehen nicht viel Sinn.

Auffassungen, die aus diesem (und anderen) Gründen Interjektionen aus dem Bereich der Sprache und damit der Sprachbetrachtung ausklammern wollen, möchte ich eine andere Konzeption entgegensetzen: Betrachtet man nämlich Sprache als offenes und dynamisches System (Hopper 1987) und berücksichtigt man die Körperlichkeit des Sprechens als immanenten Teil der Sprache, lassen sich sogenannte Randphänomene wie Interjektionen wesentlich genauer bestimmen. Letzteres habe ich versucht, am Beispiel der Lautäußerung „pff“ ansatzweise zu zeigen.

Geht man nun von einer solchen Konzeption von Sprache aus, bedeutet das auch, dass der Übergang zwischen sogenannten Naturlauten (wie einem unartiku- 
lierten Schmerzensschrei), konventionalisierten Formen (wie Quieken, Lachen, Stöhnen) und lexikalisierten Formen (wie „au“ oder „ii“) fließend ist und dass es verschiedene Zwischenformen gibt. Die Sprachwissenschaft kann meines Erachtens nur gewinnen, wenn sie eine systematische Betrachtung dieser Phänomene zulässt, indem sie ihre Konzeption von Sprache überdenkt.

\section{Literatur}

Ameka, Felix (1992a): Interjections: The universal yet neglected part of speech. In: Journal of Pragmatics 18. 101-118.

Ameka, Felix (1992b): The meaning of phatic and conative interjections. In: Journal of Pragmatics 18. 245-271.

Baldauf-Quilliatre, Heike (2016): „pf“ indicating a change in orientation in French interactions. In: Journal of Pragmatics 104. 89-107.

Baldauf-Quilliatre, Heike (2017): „pf“ as stance marker in French interactions. Annual Conference of the International Pragmatics Association, Belfast.

Baldauf-Quilliatre, Heike (i.Vorb.): Lautäußerungen im Deutschen und Französischen.

Barth-Weingarten, Dagmar (2011): Response tokens in interaction - prosody, phonetics and a visual aspect of German JAJA. In: Gesprächsforschung 12. 301-370.

Brès, Jacques (1995): „Hou! Haa! Yrrââ“: interjection, exclamation, actualisation. In: Fait de langues 6. 81-91.

Bussmann, Hadumod (1990): Lexikon der Sprachwissenschaft. (= Kröners Taschenausgabe 452). Stuttgart: Kröner.

Darwin, Charles (1873): The Expression of the Emotion in Man and Animals. London: John Murray.

Ehlich, Konrad (1986): Interjektionen. (= Linguistische Arbeiten 111). Tübingen: Niemeyer.

Ehlich, Konrad (2007): Interjektionen und Responsive. In: Hoffmann, Ludger (Hg.): Deutsche Wortarten. Berlin/New York: De Guyter. 423-444.

Goddard, Cliff (2013): Interjections and Emotion (with special reference to „surprise“ and „disgust“). In: Emotion Review. 1-11.

Goffman, Erving (1981): Forms of talk. Philadelphia: University of Pennsylvania Press.

Golato, Andrea (2012): German „oh“: marking an emotion change of state. In: ROLSI 45/3. 245-268.

Goodwin, Charles (2000): Action and embodiment within situated human interaction. In: Journal of Pragmatics 32. 1489-1522.

Günthner, Susanne (2009): Konstruktionen in der kommunikativen Praxis. Zur Notwendigkeit einer interaktionalen Anreicherung konstruktionsgrammatischer Ansätze. In: Zeitschrift für Germanistische Linguistik 37. 402-426.

Hagemann, Jörg et al. (Hgg.) (2013): Pragmatischer Standard. (= Stauffenburg Linguistik 73). Tübingen: Stauffenburg.

Hoey, Elliott (2014): Sighing in interaction: somatic, semiotic and social. In: ROLSI 47/2. 175-200. 
Hopper, Paul (1987): Emergent Grammar. In: Proceedings of the Thirteenth Annual Meeting of the Berkeley Linguistics Society, Berkeley Linguistics Society. (http://linguistics.berkeley. edu/bls/proceedings.html, Stand: 30.11.17). 139-157.

Imo, Wolfgang (2013): Sprache in Interaktionen. Analysemethoden und Untersuchungsfelder. (= Linguistik - Impulse \& Tendenzen 49). Berlin/Boston: De Gruyter.

Imo, Wolfgang (2016): Dialogizität: eine Einführung. In: Zeitschrift für Germanistische Linguistik 44/3. 337-356.

Kowal, Sabine/O'Connell, Daniel (2004): Interjektionen im Gespräch. In: Zeitschrift für Semiotik 26/1-2. 85-100.

Linell, Per (2004): The written language bias in linguistics. (= Routledge advances in communication and linguistic theory 5). Linköping: University of Linköping.

Meinard, Maruszka Eve Marie (2015): Distinguishing onomatopoeias from interjections. In: Journal of Pragmatics 76. 150-168.

Müller, Friedrich Max (1862): Lectures on the Science of Language. London: Longman.

Norrick, Neal (2009): Interjections as pragmatic markers. In: Journal of Pragmatics 41. 866-891.

Nübling, Damaris (2004): Die prototypische Interjektion: ein Definitionsvorschlag. In: Zeitschrift für Semiotik 26/1-2. 11-46.

Pomerantz, Anita (1988): Offering a candidate answer: an information seeking strategy. In: Communication Monographs 55/4. 360-373.

Pomerantz, Anita (2012): Fragen mit Antwortangebot, soziales Handeln und moralische Ordnung. In: Ayass, Ruth/Meyer, Christian (Hgg.): Sozialität in Slow Motion. Theoretische und empirische Perspektiven. Wiesbaden: Springer. 333-351.

Pompino-Marschall, Bernd (2004): Zwischen Tierlaut und sprachlicher Artikulation: Zur Phonetik der Interjektionen. In: Zeitschrift für Semiotik 26/1-2. 71-84.

Reber, Elisabeth (2012): Affectivity in Interaction. (= Pragmatics \& Beyond 215). Amsterdam/ Philadelphia: Benjamins.

Reber, Elisabeth/Couper-Kuhlen, Elisabeth (2010): Interjektionen zwischen Lexikon und Vokalität: Lexem oder Lautobjekt? In: Deppermann, Arnulf/Linke, Angelika (Hgg.): Sprache intermedial: Stimme und Schrift, Bild und Ton. (= Jahrbuch des Instituts für Deutsche Sprache 2009). Berlin/New York: De Gruyter. 69-96.

Schegloff, Emmanuel (1996): Turn organization: one intersection of grammar and interaction. In: Ochs, Elinor et al. (Hgg.): Interaction and grammar. (= Studies in interactional sociolinguistics 13). Cambridge: Cambridge University Press. 52-133.

Schmidt, Thomas/Schütte, Wilfried/Winterscheid, Jenny (2015): cGAT: Konventionen für das computergestützte Transkribieren in Anlehnung an das Gesprächsanalytische Transkriptionssystem GAT2. (http://agd.ids-mannheim.de/download/cgat_handbuch_ version_1_0.pdf, Stand: 23.5.2017).

Selting, Margret (2007): ,Grammatik des gesprochenen Deutsch' im Rahmen der Interaktionalen Linguistik. In: Ágel, Vilmos/Henning, Mathilde (Hgg.): Zugänge zur Grammatik der gesprochenen Sprache. (= Reihe Germanistische Linguistik 269). Tübingen: Niemeyer. 99-135.

Selting, Margret/Couper-Kuhlen, Elisabeth (2000): Argumente für die Entwicklung einer Interaktionalen Linguistik. In: Gesprächsforschung 1. 76-95.

Stange, Ulrike (2016): Emotive Interjections in British English. A corpus-based study on variation in acquisition, function and usage. Amsterdam/Philadelphia: Benjamins.

Trabant, Jürgen (1983): Gehören die Interjektionen zur Sprache? In: Weydt, Harald (Hg.): Partikeln und Interaktion. (= Reihe Germanistische Linguistik 44). Tübingen: Niemeyer. 69-91. 
Traverso, Véronique (2009): The dilemma of third-party complaints in conversations between friends. In: Journal of Pragmatics 41. 2385-2399.

Trojan, Felix (1948): Der Ausdruck von Stimme und Sprache. Wien: Maudrich.

Trubetzkoy, Nikolai (1939/1968): Grundzüge der Phonologie. Göttingen: Vandenhoek und Ruprecht.

Wharton, Tim (2003): Interjections, language and the ,showing/saying' continuum. In:

Pragmatics \& Cognition 11/1. 39-91.

Wilkins, David (1992): Interjections as deixis. In: Journal of Pragmatics 18. 119-158.

Wundt, Wilhelm (1911): Völkerpsychologie. Eine Untersuchung der Entwicklungsgesetze von

Sprache, Mythos und Sitte. Bd. 1: Die Sprache. Leipzig: Wilhelm Engelmann.

\section{Transkriptionskonventionen (nach GAT2)}

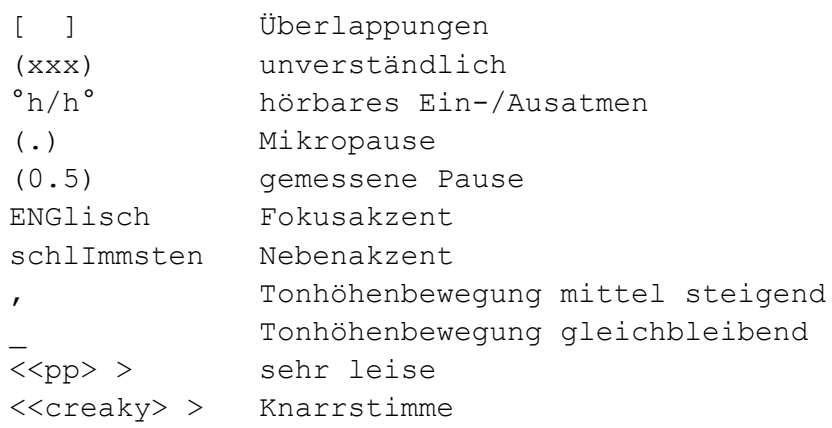


